\title{
Spin-dependent Seebeck effects in graphene-based molecular junctions
}

\author{
Jianwei Li, ${ }^{1}$ Bin Wang, ${ }^{1, *}$ Fuming Xu, ${ }^{1}$ Yadong Wei, ${ }^{1}$ and Jian Wang ${ }^{2}$ \\ ${ }^{1}$ Department of Physics, Shenzhen University, Shenzhen, Guangdong, China \\ ${ }^{2}$ Department of Physics, University of Hong Kong, Pokfulam Road, Hong Kong, China \\ (Received 23 December 2015; revised manuscript received 27 April 2016; published 18 May 2016)
}

\begin{abstract}
We report a first-principles investigation of spin-dependent transport properties in two different graphene-based molecular junctions. By applying different temperatures between two leads without bias voltage, spin-dependent currents are driven which depend on reference temperature $T$, temperature gradient $\Delta T$, and gate voltage $V_{g}$. Moreover, pure spin currents without charge currents can be obtained by adjusting $T, \Delta T$, and $V_{g}$ for both molecular junctions. The directions of pure spin currents in these two molecular junctions are opposite, which can be understood by analyzing the transmission coefficients under equilibrium states. Spin thermopower, thermal conductance, and the figure of merit as functions of $T, V_{g}$, and chemical potential $\mu$ were also investigated in the linear response regime. Large spin thermopower and spin figure of merit can be obtained by adjusting $V_{g}$ and $\mu$ for each junction, which indicates proper application of spin caloritronic devices of our graphene-based molecular junctions.
\end{abstract}

DOI: 10.1103/PhysRevB.93.195426

\section{INTRODUCTION}

The thermoelectric effect describes the direct conversion from temperature difference to electric bias and vice versa. It has received great attention in recent years with the development of electronics and spintronics. Lots of works have been carried out to explore the fundamental physics and potential applications of thermoelectric phenomena [1-3]. With the size of electronic devices shrinking to the molecular level, heat dissipation becomes aggravating due to high energy consumption. It not only dramatically depresses efficiency and performance of electronic devices, but also induces enormous energy dissipation [4]. The thermoelectric effect offers a viable solution to solve this critical problem by transferring the dissipating heat to electric energy directly. On the other hand, spintronics provides another type of feasibility to reduce energy dissipation in nanoscale devices by manipulating spin instead of charge $[5,6]$. Recently, a new research field named spin caloritronics has emerged which is based on the combination of thermoelectric effect and spintronics [7,8]. Many investigations in this area indicated that spin caloritronics is likely to be a feasible scheme to realize low power consumption in nanoscale electronics [8-11].

Graphene is one of the most exciting materials today due to its extraordinary physical and material properties. It has received extensive attention since its fabrication in 2004 [12,13]. Due to outstanding thermal and electronic performance, graphene has been recognized as one of the most excellent candidates in device fabrication of spin caloritronics. Many experimental and theoretical investigations have been carried out to detect the thermally induced spin transport properties in graphene [14-24]. Zuev et al. measured the thermoelectric properties of mesoscopic graphene versus gate voltage and explored the sign inversion behavior of thermopower (Seebeck coefficient) at the charge neutrality point [14]. Kim and co-workers deposited graphene on B-N substrates to suppress the disorder and observed large ther-

*binwang@szu.edu.cn mopower [17]. The Seebeck coefficient and Nernst coefficient were also detected in multiprobe graphene systems and both of them show oscillating behaviors versus gate voltage $[15,16]$.

Theoretically, many investigations focus on how to achieve high efficiency of thermoelectric conversion. Rameshti et al. investigated the charge and spin Seebeck effects in magnetic graphene and indicated that pure spin currents and large spin figure of merits can be obtained by varying the spin splitting, temperature, and doping of the junction [18]. Thermally induced spin Seebeck effects in magnetic zigzag graphene nanoribbons (ZGNRs) were investigated by mean-field theory $[19,20]$ and first-principles calculation [21]. Huge thermal magnetoresistances roughly equal to $10^{4} \sim 10^{5}$ between nonmagnetic configuration and different magnetic configurations were predicted. Some investigations indicated that spin thermopower and figure of merit can be largely increased by cutting graphene to be specific configurations, such as sawtooth [22], nanowiggles [23], and rectangular rings [24]. Spin-dependent Seebeck effects have also been detected in other hexagonal honeycomb structures, such as $\alpha$-ZGNRs [25] and silicene nanoribbons [26]. Although many investigations have been carried out to investigate spin-dependent Seebeck effects in ZGNRs, few investigations were focused on spin thermoelectric transport properties in graphene-based magnetic molecular junctions. The purpose of our investigation in this paper is to explore the quantum physics in this aspect.

In this paper, we study the spin-dependent Seebeck effect in two different ZGNR-polyacetylene-ZGNR molecular junctions using first-principles calculations. For each junction, thermally induced spin-up current and spin-down current flow in the opposite direction. By adjusting reference temperature, temperature gradient, and gate voltage, pure spin current can be obtained without charge current. In addition, the direction of pure spin current in the first molecular junction is opposite to that in the second molecular junction, which gives opposite signs of spin Seebeck coefficients. By increasing the length of polyacetylene chain and adjusting the gate voltage of both junctions, large spin figures of merit can be obtained at specific chemical potentials. 
The rest of the paper is organized as follows. In Sec. II, the NEGF-DFT formalism [27] of calculating spin-resolved electric and thermoelectric transport properties is introduced. In Sec. III, the numerical results of spin-resolved transmission, thermally induced spin current, spin thermopower, and spin figure of merit are presented for two different graphene-based molecular junctions. Finally, a summary is given in Sec. IV.

\section{THEORETICAL FORMALISM}

In this section, we briefly introduce the theoretical method of spin-resolved electric and thermoelectric transport properties following the train of thought in Ref. [18]. According to the Landauer-Büttiker formula within the single-electron approximation, the spin-resolved electric current is given by [28] ( $q=1$ and $h=2 \pi$ at atomic unit)

$$
\begin{aligned}
I_{\sigma}= & -\frac{q}{h} \int_{-\infty}^{+\infty} \mathcal{T}_{\sigma}(E)\left[f_{L}\left(T_{L}, \mu_{L}\left(T_{L}\right)\right)\right. \\
& \left.-f_{R}\left(T_{R}, \mu_{R}\left(T_{R}\right)\right)\right] d E .
\end{aligned}
$$

$\mathcal{T}_{\sigma}(E)$ is the spin-resolved transmission coefficient with $\sigma=\uparrow$ or $\downarrow$ indicating the spin index, which can be calculated by [29]

$$
\mathcal{T}_{\sigma}=\operatorname{Tr}\left[\Gamma_{L} G^{r} \Gamma_{R} G^{a}\right]_{\sigma \sigma} .
$$

Here, $G^{r}$ and $G^{a}$ are retarded and advanced nonequilibrium Green's functions, respectively. $\Gamma_{L}$ and $\Gamma_{R}$ are the linewidth functions which describe the coupling between leads and scattering region and $\mu_{\alpha}$ is the chemical potential of lead $\alpha$. For a spin-resolved system without spin-orbital interaction, it is natural to treat the spin-up channel and spin-down channel separately. $f_{\alpha}=\left\{1+\exp \left[\left(E-\mu_{\alpha}\right) / k_{B} T_{\alpha}\right]\right\}^{-1}$ is the Fermi distribution function of lead $\alpha$ with $\alpha=L, R$, where $T_{\alpha}$ is the temperature of lead $\alpha$ and $\mu_{\alpha}\left(T_{\alpha}\right)$ is the temperature-dependent chemical potential of lead $\alpha$.

According to Eq. (1), once a temperature gradient $\Delta T=$ $T_{R}-T_{L}$ is applied between two leads, $f_{L}-f_{R}$ is no longer zero and a spin-polarized current $I_{\sigma}$ is generated with magnitude depending on $\Delta T$ and $T_{\alpha}$. The charge current and the spin current are naturally given by

$$
I_{\mathrm{ch}}=I_{\uparrow}+I_{\downarrow}, I_{\mathrm{sp}}=\frac{\hbar}{2 e}\left(I_{\uparrow}-I_{\downarrow}\right) .
$$

Spin resolved thermopower measures the bias voltage $\Delta V$ needed to balance the spin-polarized electric current caused by temperature gradient $\Delta T$. In the linear response regime where $|\Delta V| \ll V_{\alpha}$ and $|\Delta T| \ll T_{\alpha}$, spin-resolved thermopower and thermal conductance can be obtained by [30]

$$
S_{\sigma}(\mu, T)=-\frac{1}{e T} \frac{K_{\sigma, 1}(\mu, T)}{K_{\sigma, 0}(\mu, T)}
$$

and

$$
\kappa_{\sigma}(\mu, T)=\frac{1}{h T}\left[K_{\sigma, 2}(\mu, T)-\frac{K_{\sigma, 1}^{2}(\mu, T)}{K_{\sigma, 0}(\mu, T)}\right],
$$

where $T=T_{L} \approx T_{R}$ and $\mu=\mu_{L} \approx \mu_{R} \cdot K_{\sigma, n}(\mu, T)$ is defined as

$$
K_{\sigma, n}(\mu, T)=-\int_{-\infty}^{+\infty}(E-\mu)^{n} \frac{\partial f(E, \mu, T)}{\partial E} \mathcal{T}_{\sigma}(E) d E
$$

The figure of merit describes the thermoelectric conversion efficiency of a material. For a magnetic system, the concept of the figure of merit was generalized in spin caloritronics to describe the conversion efficiency from heat to spin voltage accompanied by a spin current. According to Ref. [18], the charge figure of merit and spin figure of merit are defined as

$$
Z_{\mathrm{ch}(\mathrm{sp})} T=\frac{S_{\mathrm{ch}(\mathrm{sp})}^{2} G_{\mathrm{ch}(\mathrm{sp})} T}{\kappa} .
$$

Here $G_{\mathrm{ch}}=G_{\uparrow}+G_{\downarrow}$ and $G_{\mathrm{sp}}=\left|G_{\uparrow}-G_{\downarrow}\right|$ are charge conductance and spin conductance, respectively. $S_{\mathrm{ch}}=\left(S_{\uparrow}+\right.$ $\left.S_{\downarrow}\right) / 2$ and $S_{\mathrm{sp}}=S_{\uparrow}-S_{\downarrow}$ are charge thermopower and spin thermopower, respectively. $\kappa=\kappa_{\uparrow}+\kappa_{\downarrow}$ is charge thermal conductance.

Note that Eq. (4) and Eq. (5) are only valid at small temperature gradient and small bias voltage, where $S_{\sigma}$ and $\kappa_{\sigma}$ are expressed as a function of transmission coefficient $\mathcal{T}_{\sigma}$. In addition, although Eq. (4) and Eq. (5) are derived from the single-electron approximation where the many-body interaction is not considered, the interaction effects are partly included in the transmission coefficient $\mathcal{T}_{\sigma}$ where the Coulomb potential is obtained self-consistently from first principles [27].

\section{RESULTS AND DISCUSSION}

Figures 1(a) and 1(b) show two schematic molecular junctions in our investigation where a polyacetylene chain with finite length $N$ is sandwiched between two semiinfinite ZGNRs by different connecting formations, which are hereafter denoted as configuration I and configuration II, respectively. In configuration I, a polyacetylene chain is connected to the middle stretching carbon atomic rings of ZGNRs, while it bridges the side carbon rings in configuration II. As will be illustrated in the following, the small difference of connecting formation in the two configurations produces
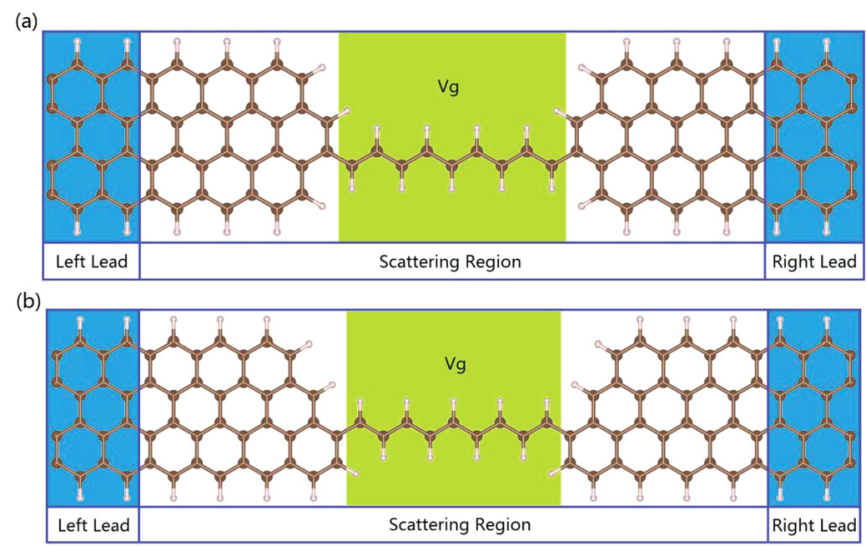

FIG. 1. Schematic structures of ZGNR-polyacetylene-ZGNR molecular junctions where a polyacetylene chain is sandwiched between two semi-infinite ZGNRs by different connecting formation as illustrated in (a) and (b). In (a), a polyacetylene chain with length $N=9$ is connected to the middle stretching carbon atomic rings of ZGNRs with width $M=4$. In (b), a polyacetylene chain with length $N=9$ bridges the side carbon atomic rings of ZGNRs with $M=4$. ZGNRs are extended to the left and the right infinity which are highlighted by blue color. A gate voltage is supplied in the region of the polyacetylene chain which is also highlighted. 
opposite flowing direction of thermally induced spin current. To keep the symmetry of structures, only the polyacetylene chains with length equal to odd numbers $(N=7,9,11,13)$ are considered for both configurations. In our investigation, we assume each ZGNR lead is in the ferromagnetic (FM) state with parallel magnetic moments on both edges, because the antiferromagnetic (AFM) formation of ZGNRs shows semiconducting behavior [31]. We also assume that the orientation of the net magnetic moments in the left lead is parallel to that in the right lead to obtain a nonzero transmission coefficient. In addition, although the AFM formation of ZGNRs is more stable [32], FM formation is also easily realized by external magnetic field considering the slight difference of energy per edge atom $\sim 2 \mathrm{meV}$ between FM state and AFM state [33].

The geometric optimization and spin electronic transport investigation are implemented using the first-principles package NanoDcal [34], which is based on the state-of-the-art NEGF-DFT method [27]. In our calculation, norm-conserving pseudopotentials are used to define atomic cores [35] and exchange correlation is treated at the LSDA level [36]. A conjugate gradient method is used to achieve total-energy minimization until the residual force on each atom is less than $0.05 \mathrm{eV} / \AA$. A single-zeta basis set is employed to solve the Kohn-Sham equation self-consistently until the convergence criterion of energy is less than $10^{-5}$ a.u. [37]. Finally, the Hamiltonian including all the interactions of the system as well as the effect of external fields is obtained.

In the following, we present the numerical results from first principles about the spin-resolved electric and thermal electric transport behaviors of both configurations.

\section{A. Spin-resolved electric transport}

In this section, we report the spin-resolved electric transport behaviors for both configurations. Figure 2 shows the spinresolved transmission coefficient $\mathcal{T}_{\sigma}$ as a function of energy $E$ under zero bias voltage and zero gate voltage, where panel (a) and panel (b) are for configuration I and panel (c) and panel (d) are for configuration II. For each panel, molecular junctions with different length $N$ of polyacetylene chains show similar behaviors of $\mathcal{T}_{\sigma}$. For configuration I, two peaks of transmission coefficients appear in the energy window from $-0.5 \mathrm{eV}$ to $0.5 \mathrm{eV}$ for both spin-up and spin-down channels. The peaks of $\mathcal{T}_{\uparrow}$ [see panel (a)] and $\mathcal{T}_{\downarrow}$ [see panel (b)] are roughly symmetric with respect to the Fermi level $E_{F}$. To understand the behavior of $\mathcal{T}_{\sigma}$, we plotted the spin-resolved density of states (DOS) of the ZGNR in Fig. 2(a) and Fig. 2(b) as indicated by the filled areas. It is found that the left sharp peak of $\mathcal{T}_{\uparrow}$ and the right sharp peak of $\mathcal{T}_{\downarrow}$ have large overlap with the spin-resolved edge states of the ZGNR. Further investigation indicates that these two peaks are not shifted by gate voltage applied in the molecular region. This means that the two peaks of $\mathcal{T}_{\sigma}$ are mostly contributed by the spin-resolved edge states of the ZGNR. Similar properties can be found for configuration II as shown in Fig. 2(c) and Fig. 2(d), where two sharp peaks of $\mathcal{T}_{\uparrow}$ and $\mathcal{T}_{\downarrow}$ appear at $E \approx \pm 0.3 \mathrm{eV}$ which correspond well with the spin-resolved edge states of the ZGNR.

The broad peaks of $\mathcal{T}_{\sigma}$ near the Fermi level shown in each panel of Fig. 2 can be understood by analyzing the spinresolved real-space charge distribution of both configurations. Figures 3(a) and 3(b) show the net magnetic moment (number of spin-up electrons minus the number of spin-down electrons) summed in the $x-y$ plane versus the $z$ direction, where the red
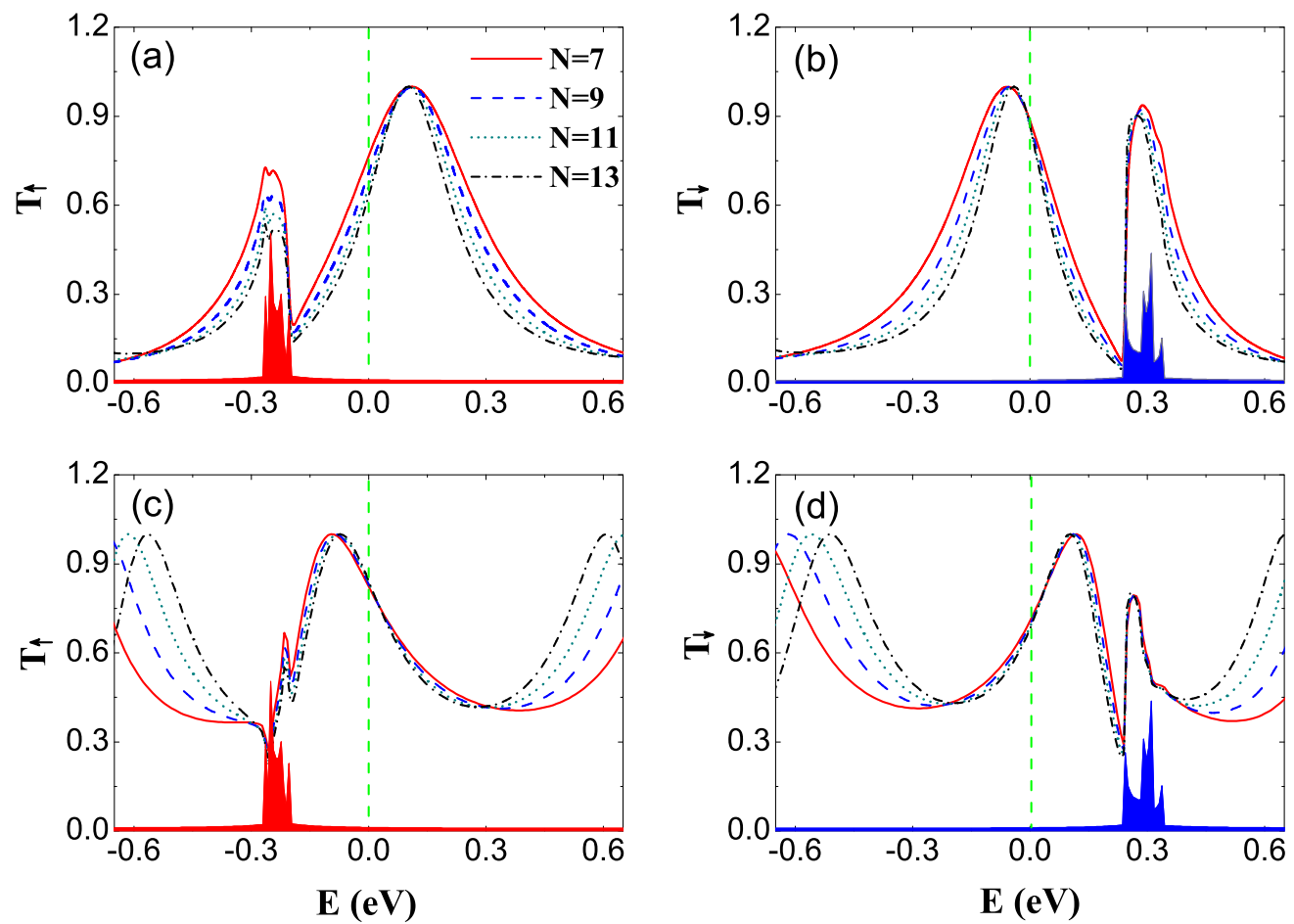

FIG. 2. (a) $\mathcal{T}_{\uparrow}$ and (b) $\mathcal{T}_{\downarrow}$ for configuration I with $N=7,9,11,13$. (c) $\mathcal{T}_{\uparrow}$ and (d) $\mathcal{T}_{\downarrow}$ for configuration II with $N=7,9,11,13$. The red filled areas in (a) and (c) indicate the spin-up edge state of ZGNR, and the blue filled areas in (b) and (d) indicate the spin-down edge state of ZGNR. The vertical green lines in each panel indicate the Fermi level $E_{F}=0$. 

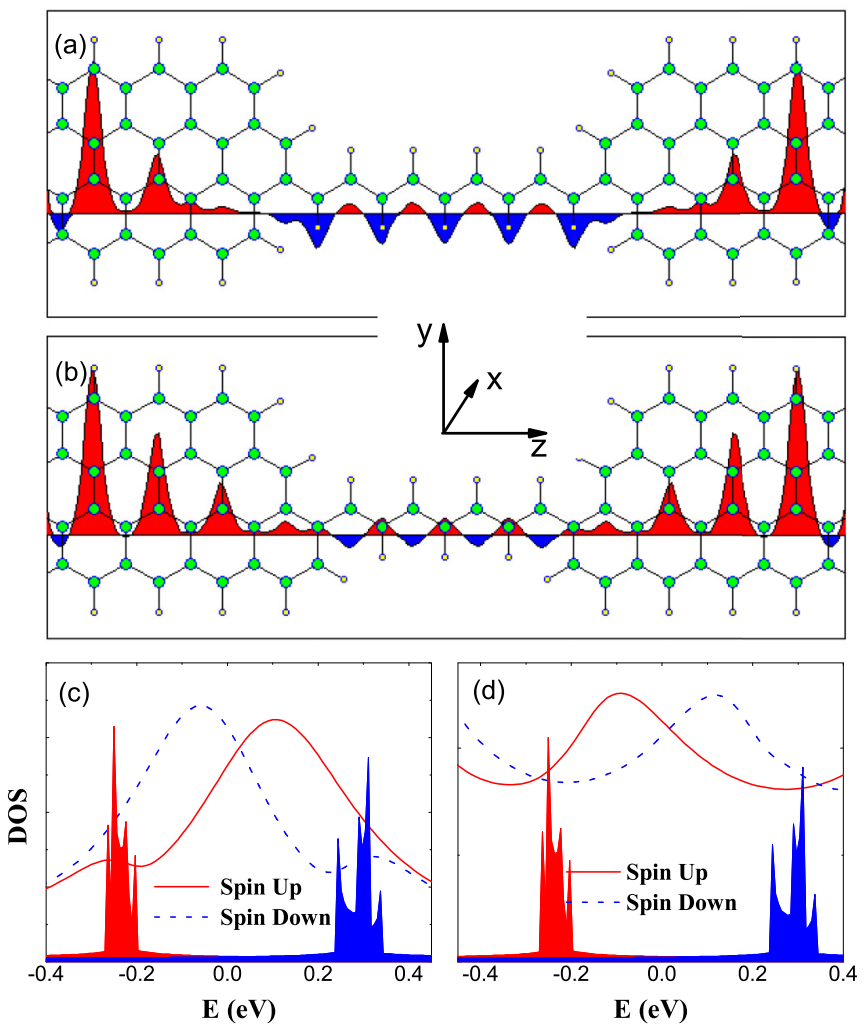

FIG. 3. (a), (b) Configurations and net magnetic moment summed in $x$-y plane versus $z$ direction for (a) configuration I and (b) configuration II. The red areas and the blue areas in (a) and (b) correspond to positive and negative net magnetic moments, respectively. (c), (d) Spin-up DOS (red curves) and spin-down DOS (blue curves) versus energy of reconfigured isolated polyacetylene chain for (c) configuration I and (d) configuration II. The red filled areas and the blue filled areas in (c) and (d) indicate the spin-up and spin-down DOS of the ZGNR, respectively.

area and the blue area correspond to positive and negative net magnetic moments, respectively. For configuration I, the net magnetic moment on polyacetylene chain is negative, which is opposite to that on ZGNRs, while for configuration II, the net magnetic moment on the polyacetylene chain is positive, which is the same as that on ZGNRs. It is the opposite orientations of the net magnetic moment on polyacetylene chains that determines the different behaviors of $\mathcal{T}_{\sigma}$ of configuration I and configuration II.

To further confirm this view, we plotted the spin-resolved DOS of a reconfigured isolated polyacetylene chain in Fig. 3(c) and Fig. 3(d) for configuration I and configuration II, respectively, where the DOS of the system is projected onto the polyacetylene chain. As a comparison, the spin-resolved DOS of the ZGNR is also shown by filled areas. For configuration I, the peak energy of the spin-up DOS of the polyacetylene chain is above the Fermi level, while the peak energy of the spin-down DOS of the polyacetylene chain is below the Fermi level, which are reversed compared to those of the ZGNR because of the opposite orientations of net magnetic moment on polyacetylene chain and ZGNRs. The peaks of the spin-resolved DOS of a polyacetylene chain correspond well to the broad peaks of $\mathcal{T}_{\sigma}$ near the Fermi level as shown in
Fig. 2(a) and Fig. 2(b). Furthermore, renormalized molecular levels (RMLs) of polyacetylene chains are greatly affected by the presence of ZGNR leads, where the corresponding part of the Hamiltonian matrix of the system is cut out to describe the isolated polyacetylene chains. By projecting the DOS of the reconfigured polyacetylene chain onto the molecular levels, we further conclude that the peaks of the spin-resolved DOS near the Fermi level are mostly contributed by the renormalized HOMO states. It is the spin-resolved HOMO states of reconfigured polyacetylene chains that determine the features of $T_{\sigma}$ near the Fermi level. For configuration II, the peak of the spin-up DOS is below the Fermi level, while the peak of the spin-down DOS is above the Fermi level as shown in Fig. 3(d), which shows consistent behaviors with DOS of ZGNRs because of the same orientation of net magnetic moment on polyacetylene chains and ZGNRs. Similarly, the peaks of the DOS of reconfigured isolated polyacetylene chains correspond well to the broad peaks of $\mathcal{T}_{\sigma}$ near the Fermi level as shown in Fig. 2(c) and Fig. 2(d).

Electric transport properties can be modulated by applying a gate voltage to the polyacetylene chain. In our numerical calculation, a uniform electric field is applied in the molecular region perpendicular to the ZGNR plane. The final effective potential due to electric field (effect of gate voltage) is determined self-consistently from first-principles calculations. Figures 4(a) and 4(b) show $\mathcal{T}_{\uparrow}$ and $\mathcal{T}_{\downarrow}$, respectively, as a function of energy $E$ at different gate voltages $V_{g}$ for configuration I. With the increase of gate voltage from $-8 \mathrm{~V}$ to $8 \mathrm{~V}$, the renormalized HOMO level of the polyacetylene chain is pushed down to lower energy. Because the resonant state of the transmission coefficient around the Fermi level is dominated by the renormalized HOMO state, the peak of $\mathcal{T}_{\sigma}$ shifts from the right side to the left side of $E_{F}$. Similar gate controlled properties of the transmission coefficient are also found for configuration II as shown in Fig. 4(d) and Fig. 4(e).

To further investigate the gate-controlled behaviors of the transmission coefficient, we plot the spin-resolved conductance $G_{\sigma}$ as functions of gate voltage for configuration I in Fig. 4(c) and configuration II in Fig. 4(f). For both configurations, $G_{\sigma}$ increases first and then decreases as a function of $V_{g}$ for all the polyacetylene chains with different length. $G_{\sigma}$ can be tuned roughly from 1 to 0.1 (the unit is $e^{2} / h$ ) for configuration I and from 1 to 0.3 for configuration II, which indicates that the gate efficiency of the former is higher than that of the latter. For configuration I, $G_{\uparrow}$ reaches the maximum value under a positive gate voltage, while $G_{\downarrow}$ reaches the maximum value under a negative gate voltage. The tendency of $G_{\uparrow}\left(G_{\downarrow}\right)$ as a function of $V_{g}$ for configuration II is similar to that of $G_{\downarrow}\left(G_{\uparrow}\right)$ for configuration I. This gate-controlled behavior of $G_{\sigma}$ is accordant with the information given by Fig. 2.

\section{B. Thermally induced current}

In this section, we study the thermally induced spinresolved currents for configuration I and configuration II. Because the length of the polyacetylene chain hardly changes the electric transport behaviors for both configurations as shown in Fig. 2, we only investigate the thermally driven spin current for the structures with $N=9$ for configuration I and 

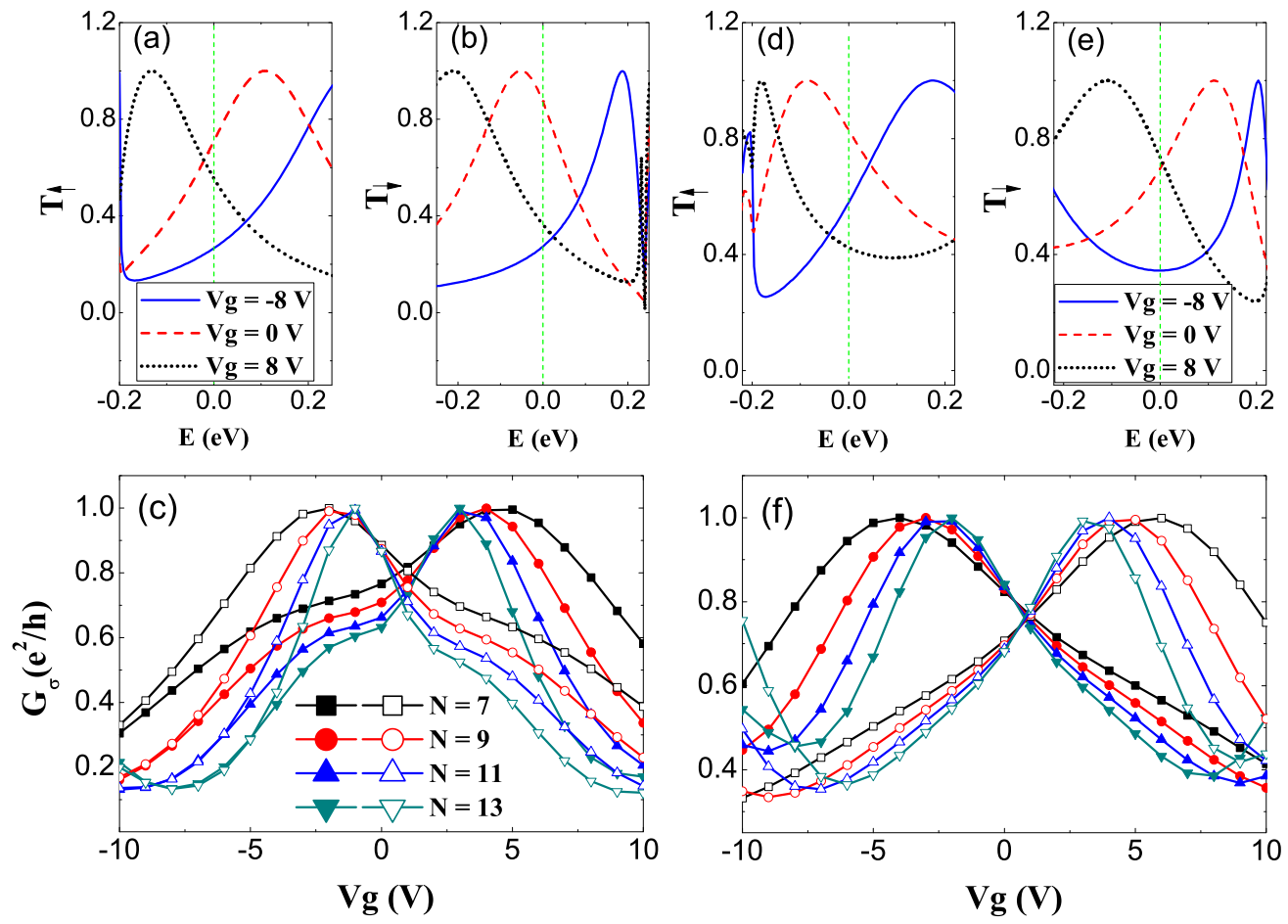

FIG. 4. (a) $\mathcal{T}_{\uparrow}$ and (b) $\mathcal{T}_{\downarrow}$ as functions of energy under different gate voltage for configuration I with $N=9$. (c) $G_{\sigma}$ as a function of gate voltage for configuration I. (d) $\mathcal{T}_{\uparrow}$ and (e) $\mathcal{T}_{\downarrow}$ as functions of energy under different gate voltage for configuration II with $N=7$. (f) $G_{\sigma}$ as a function of gate voltage for configuration II. In (a), (b), (d), and (e), the vertical dashed lines indicate the Fermi level. In (c) and (f), the solid symbol curves indicate $G_{\uparrow}$ and the open symbol curves indicate $G_{\downarrow}$.

$N=7$ for configuration II. In our calculation, the chemical potentials of both leads were shifted to zero initially when temperature is equal to zero, i.e., $\mu_{L}=\mu_{R}=0$. Theoretically, $\mu_{L}$ and $\mu_{R}$ should be changed to keep charge conservation with increasing of temperature [18]. However, we found that the shift of chemical potential is only roughly $1 \mathrm{meV}$ when temperature is increased from zero to $200 \mathrm{~K}$, which is too small to influence the thermally induced current according to Eq. (1). Therefore, we ignored the temperature influence of the chemical potential in current calculations and always set $\mu_{L}=\mu_{R}=\mu$.

When a temperature gradient $\Delta T$ is applied between the left lead and the right lead, $f_{L}(T)-f_{R}(T+\Delta T)$ is no longer equal to zero and shows inverse symmetric behaviors around chemical potential $\mu$. For a given $T$, the magnitude of $f_{L}-f_{R}$ increases versus $\Delta T$ as shown in Fig. 5(a), while for a given $\Delta T$, the magnitude of $f_{L}-f_{R}$ decreases and its width increases versus $T$ as shown in Fig. 5(b). Figures 5(c) and 5(d) show the spin-resolved currents $I_{\sigma}$ as functions of $\Delta T$ at different $T$ for configuration I and configuration II, respectively. For both configurations, $I_{\sigma}$ shows roughly linear behavior versus $\Delta T$ because $f_{L}-f_{R}$ is proportional to $\Delta T$ when $\Delta T \ll T$ [18]. Because $f_{L}-f_{R}$ is an odd function, the sign of $I_{\sigma}$ is determined by the slope of the transmission coefficient $\mathcal{T}_{\sigma}$ near the Fermi level according to Eq. (1). For configuration I, $I_{\uparrow}$ is positive and $I_{\downarrow}$ is negative at different $T$ because $\mathcal{T}_{\uparrow}$ increases and $\mathcal{T}_{\downarrow}$ decreases as functions of energy near the Fermi level. Positive values of $I_{\sigma}$ indicate accordant directions of $I_{\sigma}$ and temperature gradient, and vice versa. For configuration II, $I_{\uparrow}$ is negative and $I_{\downarrow}$ is positive at different $T$ because $\mathcal{T}_{\uparrow}$ decreases and $\mathcal{T}_{\downarrow}$ increases as functions of energy near the Fermi level, which is opposite to that shown in configuration I. Figures 5(e) and 5(f) show the spin-resolved currents $I_{\sigma}$ as functions of $T$ at different $\Delta T$ for configuration I and configuration II, respectively. For both configurations, $I_{\sigma}$ increases as a function of $T$ because the width of $f_{L}-f_{R}$ is broadened around the Fermi level with increase of $T$, although its amplitude decreases as shown in Fig. 5(b). In addition, $I_{\sigma}$ is proportional to $\Delta T$, which is accordant with that shown in Fig. 5(c) and Fig. 5(d). For configuration I, $I_{\uparrow}$ is positive and $I_{\downarrow}$ is negative, and therefore $I_{\mathrm{sp}}$ is positive, while for configuration II, $I_{\mathrm{sp}}$ is always negative.

More interestingly, pure spin current without charge current can be obtained by adjusting $T, \Delta T$, and $V_{g}$ for both configurations. Figures 6(a) and 6(b) show the thermally induced currents, including $I_{\sigma}, I_{\mathrm{ch}}$, and $I_{\mathrm{sp}}$, as functions of gate voltage at $T=120 \mathrm{~K}$ and $\Delta T=5 \mathrm{~K}$ for configuration I and configuration II, respectively. For configuration I, $I_{\uparrow}$ increases slowly and then decreases rapidly to negative, while $I_{\downarrow}$ decreases to negative first and then increases slowly. As a result, $I_{\mathrm{ch}}$ decreases from positive to negative and $I_{\mathrm{sp}}$ increases first and then decreases. Especially, a positive pure spin current $I_{\mathrm{sp}}=4.7 \hbar / 2 e \mathrm{nA}$ without charge current occurs when $V_{g}=0$. For configuration II, a pure current can also be obtained when $V_{g}=3.6 \mathrm{~V}$ although its sign is negative. To further explore how a pure spin current can be achieved by tuning the knobs, e.g., gate voltage, temperature, and temperature difference, we scanned $T$ and $\Delta T$ under different $V_{g}$ for both configurations to obtain the pure spin current. The results are shown in Fig. 6(c) and Fig. 6(d), where each curve indicates the appearance of pure spin current with different colors indicating the magnitude of $I_{\mathrm{sp}}$. For configuration I, pure spin current 

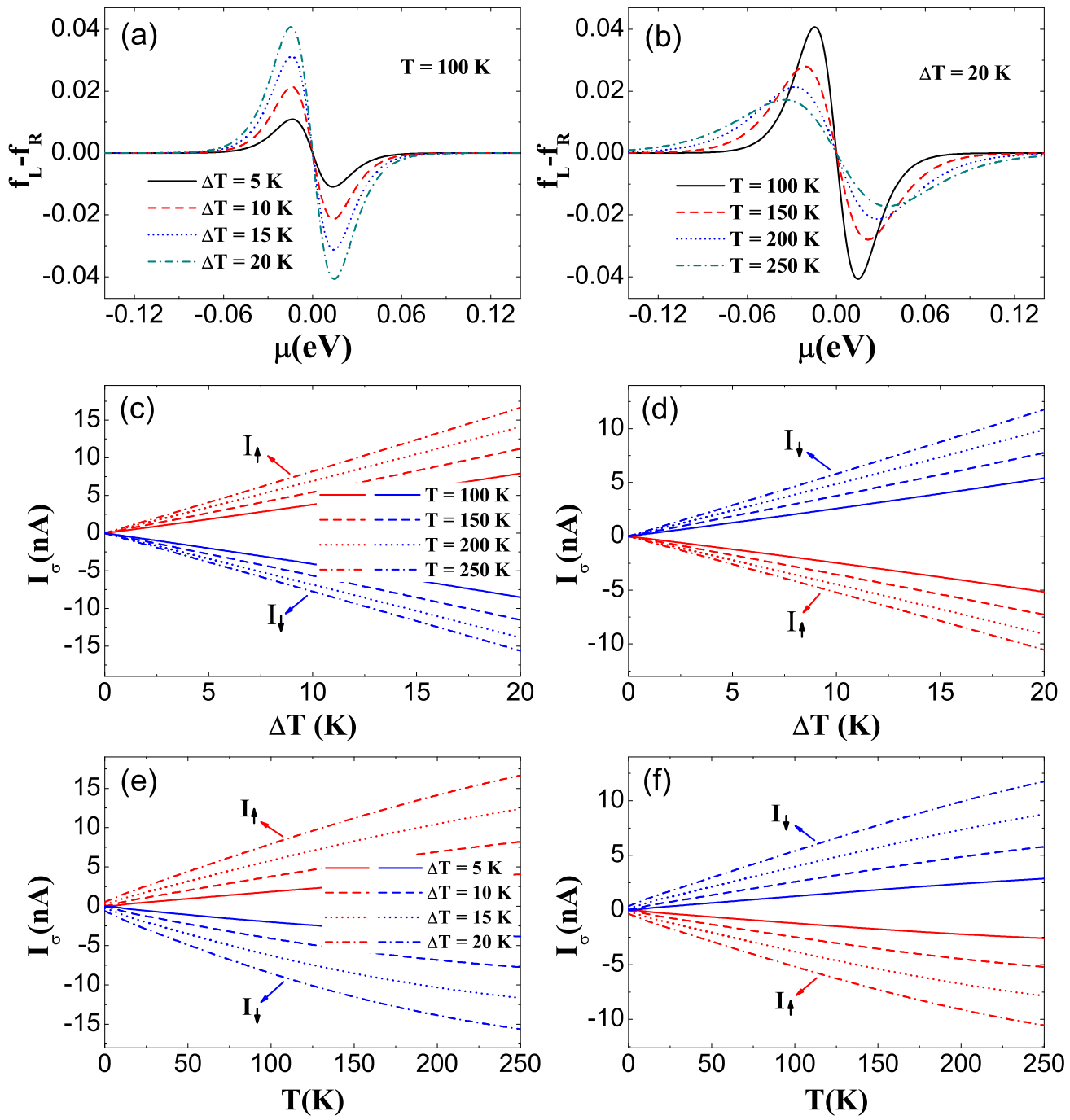

FIG. 5. (a) $f_{L}(T)-f_{R}(T+\Delta T)$ as a function of $\mu$ at finite $T$ and different $\Delta T$. (b) $f_{L}(T)-f_{R}(T+\Delta T)$ as a function of $\mu$ at finite $\Delta T$ and different $T$. (c) and (d) $I_{\sigma}$ as a function of $\Delta T$ at different $T$. (e) and (f) $I_{\sigma}$ as a function of $T$ at different $\Delta T$. Panel (c) and panel (e) are for configuration I with $N=9$. Panel (d) and panel (f) are for configuration II with $N=7$.

can be achieved from $V_{g}=-0.2 \mathrm{~V}$ to $0.2 \mathrm{~V}$ at small $\Delta T$ and corresponding $T$, where $T$ and $\Delta T$ show roughly linear behavior. Considering the nonconsecutive change of $V_{g}$ in our first-principles calculation, we predict that pure spin current can always be achieved in the area framed by $T, \Delta T$, and $V_{g}$ as indicated by the parallelogram in Fig. 6(c). For a specific $T$ in this area, the magnitude of pure spin current increases versus $\Delta T$ accompanied with decrease of $V_{g}$, while for a given $\Delta T$, the magnitude of pure spin current increases versus $T$ accompanied with decrease of $V_{g}$. Similar results can also be found for configuration II, where pure current can be achieved under different $T, \Delta T$, and $V_{g}$ framed by the parallelogram as shown in Fig. 6(d).

\section{Thermopower and thermal conductance}

To obtain more information on spin-resolved thermal transport, we calculated thermopower and thermal conductance of both configurations using Eq. (4) and Eq. (5). Figure 7 shows $S_{\mathrm{ch}}, S_{\mathrm{sp}}$, and $\kappa_{\sigma}$ as functions of temperature $T$ at the Fermi level $E_{F}=0 . S_{\mathrm{ch}}, S_{\mathrm{sp}}$, and $\kappa_{\sigma}$ increase almost linearly with $T$ for both configurations with different $N$ when $T$ is less than $150 \mathrm{~K} . S_{\mathrm{sp}}$ is negative for configuration I and positive for configuration II, while $S_{\mathrm{ch}}$ is very close to zero for both configurations. Because $S_{\text {ch }}=\left(S_{\uparrow}+S_{\downarrow}\right) / 2$ and $S_{\mathrm{sp}}=S_{\uparrow}-S_{\downarrow}$, the low-temperature behaviors of $S_{\mathrm{ch}}$ and $S_{\mathrm{sp}}$ can be understood by expanding Eq. (4) to the first order of temperature as follows,

$$
\begin{aligned}
S_{\sigma}(\mu, T)= & -\frac{\pi^{2} k_{B}^{2} T}{3 e}\left[\frac{\partial \ln \left[G_{\sigma}(\mu)\right]}{\partial \mu}+\frac{\pi^{2} k_{B}^{2} T^{2}}{15 G_{\sigma}(\mu)} \frac{\partial^{3} G_{\sigma}(\mu)}{\partial \mu^{3}}\right] \\
& +\cdots
\end{aligned}
$$

Similarly, $\kappa_{\sigma}$ in Eq. (5) can also be expanded at low temperature, where

$$
\begin{aligned}
\kappa_{\sigma}(\mu, T)= & \frac{\pi^{2} k_{B}^{2} T}{3 e^{2}} G_{\sigma}(\mu)\left\{1+\frac{\pi^{2} k_{B}^{2} T^{2}}{15}\left[\frac{8}{G_{\sigma}(\mu)} \frac{\partial^{2} G_{\sigma}(\mu)}{\partial \mu^{2}}\right.\right. \\
& \left.\left.-5\left(\frac{\partial \ln \left[G_{\sigma}(\mu)\right]}{\partial \mu}\right)^{2}\right]\right\}+\cdots
\end{aligned}
$$



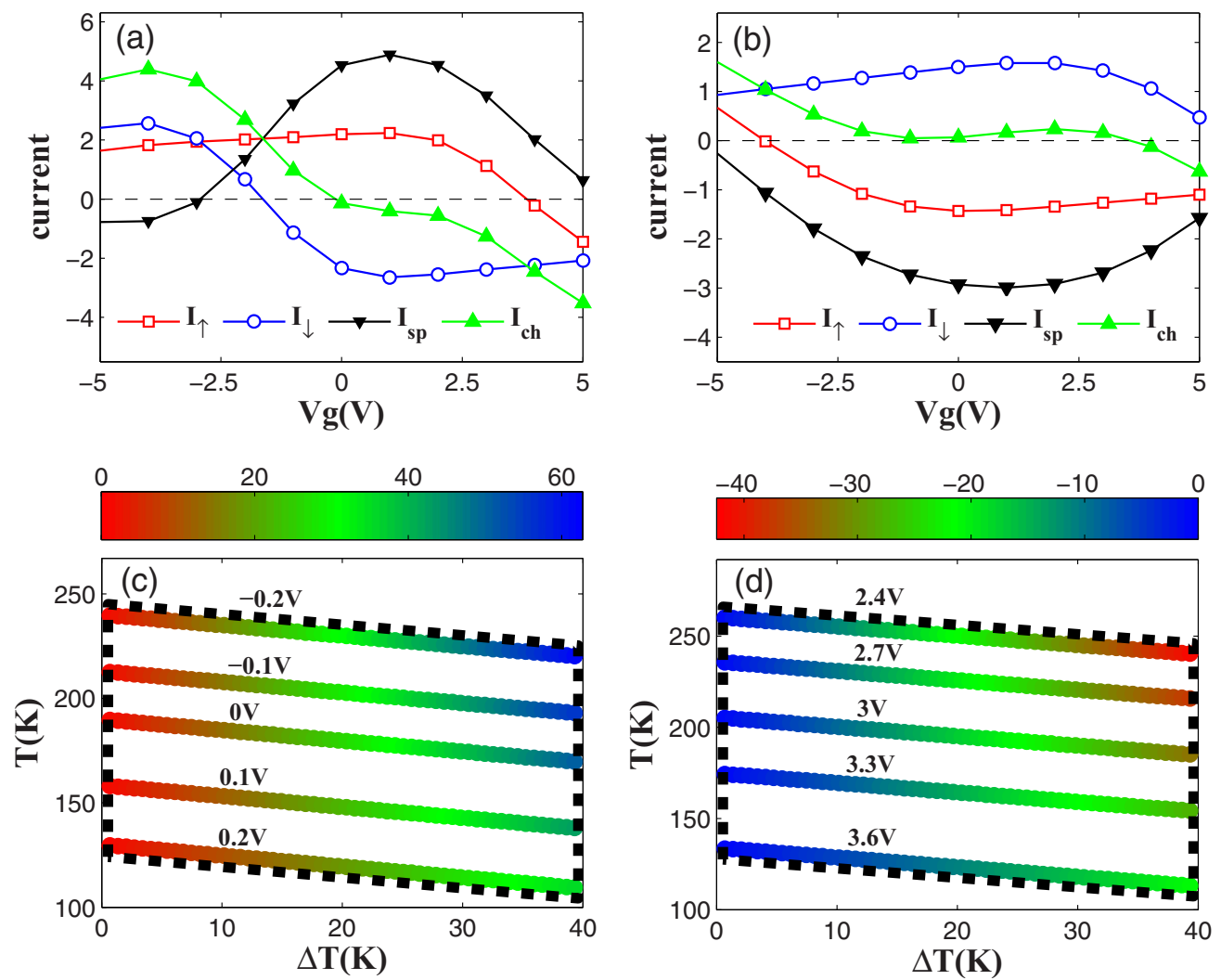

FIG. 6. (a) and (b) $I_{\uparrow}, I_{\downarrow}, I_{\mathrm{ch}}$, and $I_{\mathrm{sp}}$ as functions of gate voltage with $T=120 \mathrm{~K}$ and $\Delta T=5 \mathrm{~K}$. (c) and (d) Pure spin current versus $T$ and $\Delta T$ under different gate voltage. The different colors of each curve indicate the magnitudes of pure spin current. Panel (a) and panel (c) are for configuration I with $N=9$. Panel (b) and (d) are for configuration II with $N=7$. The unit of $I_{\sigma}$ and $I_{\mathrm{ch}}$ is nA, and of $I_{\mathrm{sp}}$ is $\hbar / 2 e \mathrm{nA}$.
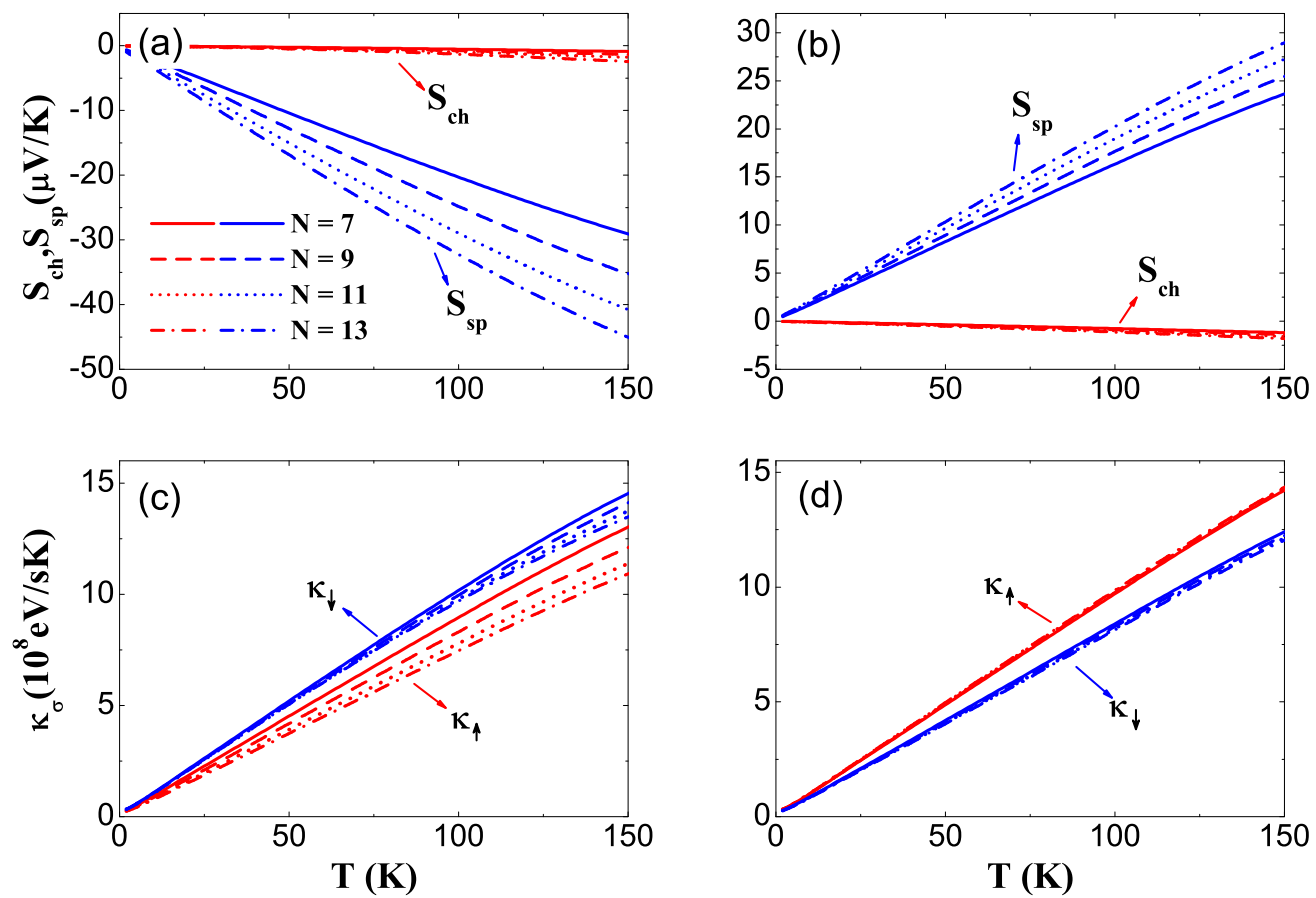

FIG. 7. (a), (b) $S_{\mathrm{ch}}$ (red curves), $S_{\mathrm{sp}}$ (blue curves) and (c), (d) $\kappa_{\uparrow}$ (red curves) and $\kappa_{\downarrow}$ (blue curves) as functions of $T$ at $\mu=0$ for $N=7$ (solid curves), $N=9$ (dashed curves), $N=11$ (dotted curves), and $N=13$ (dash-dotted curves). Panel (a) and panel (c) are for configuration I, and panel (b) and panel (d) are for configuration II. 

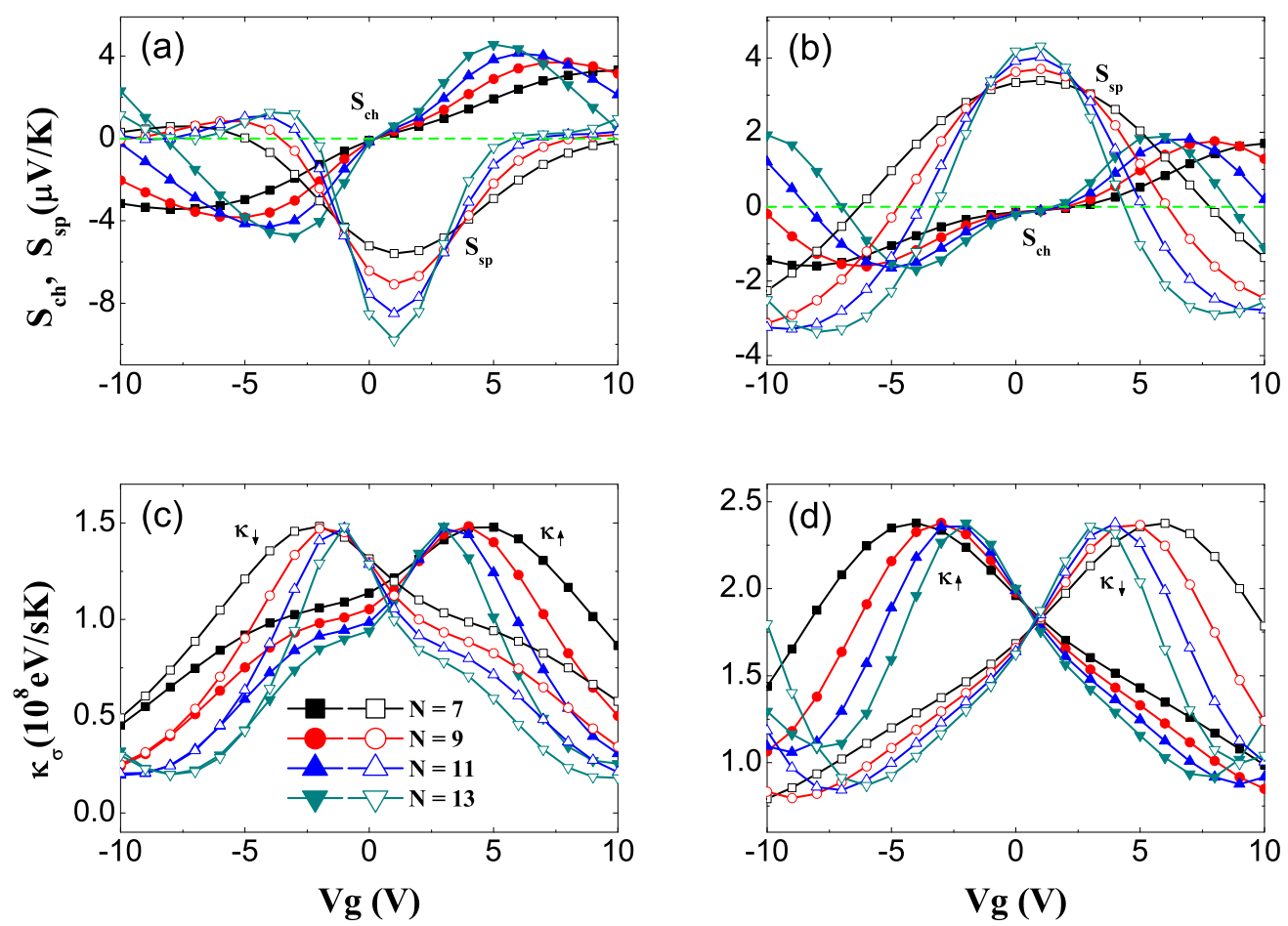

FIG. 8. (a), (b) Thermopower and (c), (d) thermal conductance as functions of gate voltage at $T=20$ K. Panels (a) and (c) are for configuration I with $N=7,9,11,13$. Panels (b) and (d) are for configuration II with $N=7,9,11,13$. In panels (a) and (b), the solid symbol curves indicate $S_{\mathrm{ch}}$, while the open symbol curves indicate $S_{\mathrm{sp}}$. In panels (c) and (d), the solid symbol curves indicate $\kappa_{\uparrow}$, while the open symbol curves indicate $\kappa_{\downarrow}$.

Obviously, $S_{\sigma}$ and $\kappa_{\sigma}$ are proportional to the first order of temperature when $T$ is small and the sign of $S_{\sigma}$ is determined by $\partial G / \partial \mu$ at chemical potential $\mu$. For configuration $\mathrm{I}, \partial G_{\uparrow}\left(E_{F}\right) / \partial \mu$ is positive as shown in Fig. 2(a) and $\partial G_{\downarrow}\left(E_{F}\right) / \partial \mu$ is negative as shown in Fig. 2(b); therefore $S_{\uparrow}$ is negative and $S_{\downarrow}$ is positive. As a result, $S_{\mathrm{ch}}$ is roughly canceled to zero and $S_{\mathrm{sp}}$ is negative as shown in Fig. 7(a). For configuration II, $\partial G_{\uparrow}\left(E_{F}\right) / \partial \mu$ is negative as shown in Fig. 2(c) and $\partial G_{\downarrow}\left(E_{F}\right) / \partial \mu$ is positive as shown in Fig. 2(d); therefore $S_{\uparrow}$ is positive and $S_{\downarrow}$ is negative. Consequently, $S_{\text {ch }}$ is very small and $S_{\text {sp }}$ is positive as shown in Fig. 7(b). The linear relationship of thermopower versus temperature has been measured by experiment in graphene systems [14]. In addition, $\kappa_{\sigma}$ is linearly proportional to $G_{\sigma}$ according to Eq. (9), so $\kappa_{\uparrow}$ is a little smaller than $\kappa_{\downarrow}$ for configuration I because $G_{\uparrow}$ is a little smaller than $G_{\downarrow}$, while for configuration II, $\kappa_{\uparrow}$ is a little larger than $\kappa_{\downarrow}$ because $G_{\uparrow}$ is a little larger than $G_{\downarrow}$.

Figures 8(a) and 8(b) show the spin-resolved thermopower as functions of gate voltage at $T=20 \mathrm{~K}$ for configuration I and configuration II, respectively. Obviously, the gate voltage can effectively tune the magnitude and even the sign of thermopower. Configuration I and configuration II exhibit roughly opposite tendency of $S_{\mathrm{sp}}$ as a function of gate voltage which means opposite flowing directions of spin-polarized currents in the two structures. For both configurations, $\kappa_{\sigma}$ increases first and then decreases as a function of gate voltage as shown in Fig. 8(c) and Fig. 8(d), which display the same behavior of $G_{\sigma}$ as shown in Fig. 3(c) and Fig. 3(d). This is reasonable because $\kappa_{\sigma}$ is proportional to $G_{\sigma}$ at low temperature as given by Eq. (9).
To improve the performance of the thermoelectric effect which is described by the figure of merit, small conductance, small thermal conductance, and large thermopower are essential according to Eq. (7). Although $S_{\mathrm{ch}}$ and $S_{\mathrm{sp}}$ can be adjusted by gate voltage as shown in Fig. 8, it is not enough to obtain a favorable $Z_{\mathrm{ch}(\mathrm{sp})} T$ at the Fermi level. By changing chemical potential $\mu$ and $V_{g}$, we found that the magnitude of $Z_{\mathrm{ch}(\mathrm{sp})} T$ can be largely increased in the energy region from $-0.25 \mathrm{eV}$ to $-0.2 \mathrm{eV}$ and from $0.2 \mathrm{eV}$ to $0.25 \mathrm{eV}$, where spin-resolved edge states of ZGNRs exist. Figure 9(a) shows $\mathcal{T}_{\downarrow}$ versus energy under different gate voltages for configuration I with $N=13$. With increase of gate voltage from $0 \mathrm{~V}$ to $8 \mathrm{~V}$, the renormalized spin-down LUMO state of the reconfigured polyacetylene chain is pushed far away from the spin-down edge state of ZGNRs. As a result, an antiresonance dip of $\mathcal{T}_{\downarrow}$ appears when $V_{g}=4 \mathrm{~V}$. With further increasing of gate voltage, a gap of $\mathcal{T}_{\downarrow}$ appears near $E=0.23 \mathrm{eV}$. This gap also depends on the length of the polyacetylene chain. For a given gate voltage, the longer the polyacetylene chain, the larger is the gap as shown in Fig. 9(b). Large $Z_{\mathrm{ch}(\mathrm{sp})} T$ can be obtained at the boundary of the transmission gap due to small conductance, small thermal conductance, and rapid change of conductance. Figures 9(c) and 9(d) show $S_{\mathrm{sp}}$ and $Z_{\mathrm{sp}} T$, respectively, versus chemical potential $\mu$ for configuration I with $T=20 \mathrm{~K}$ and $N=7,9,11,13 . S_{\mathrm{sp}}$ and $Z_{\mathrm{sp}} T$ increase with the length of the polyacetylene chain accompanied by slight shift of peak values. For configuration I with $N=13$, the maximum value of $S_{\mathrm{sp}}$ can reach $\sim 300 \mu \mathrm{V} / \mathrm{K}$ and the figure of merit $Z_{\mathrm{sp}} T$ can reach $\sim 1.6$. Large $S_{\mathrm{sp}}$ and $Z_{\mathrm{sp}} T$ can also be achieved in the energy range from $-0.25 \mathrm{eV}$ to $-0.2 \mathrm{eV}$ with negative gate voltage (not shown in figures). This indicates that the 

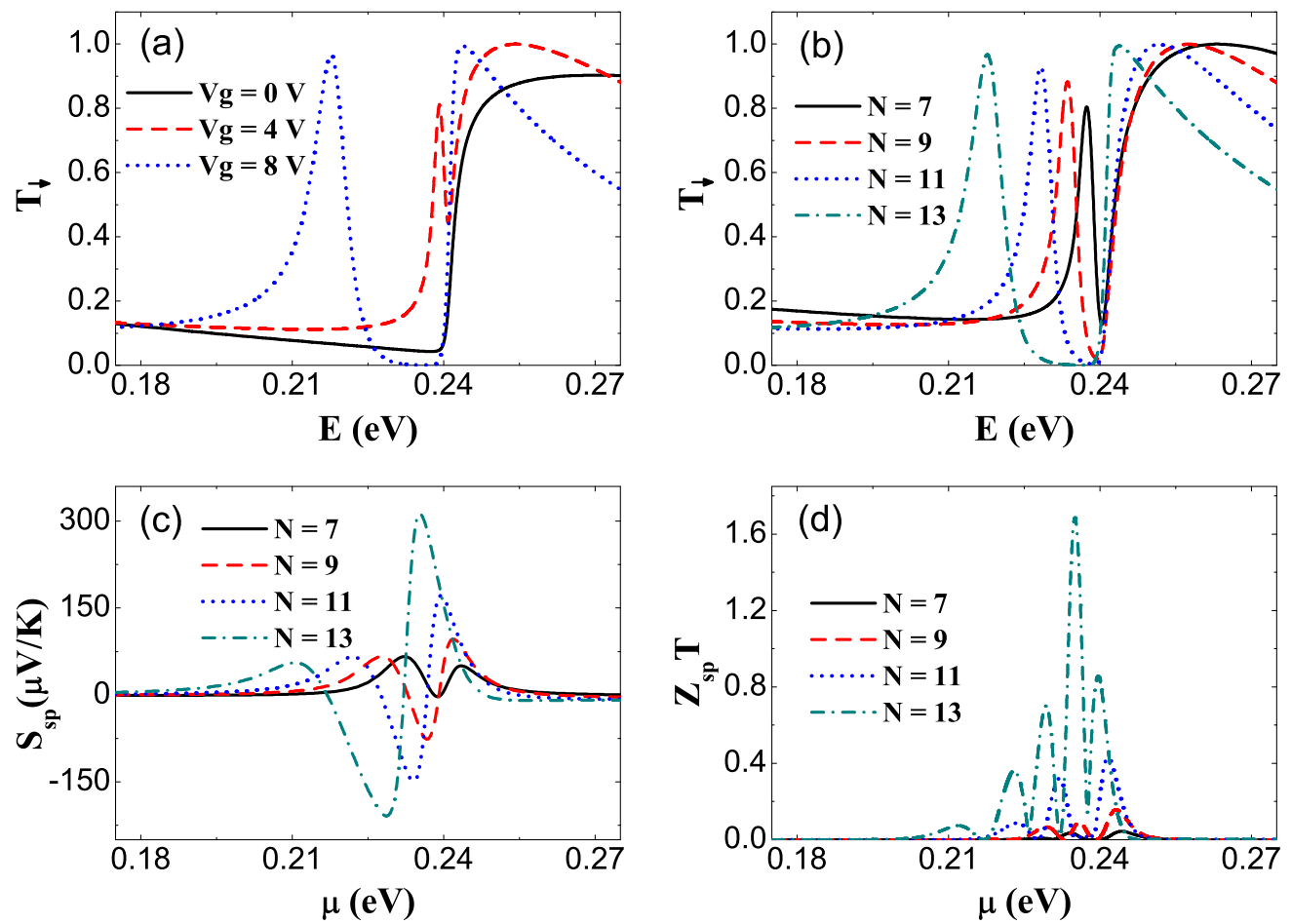

FIG. 9. (a) $\mathcal{T}_{\downarrow}$ as a function of energy under different gate voltage $V_{g}$ for configuration I with $N=13$. (b) $\mathcal{T}_{\downarrow}$ as a function of energy under $V_{g}=8 \mathrm{~V}$ for configuration I with different $N$. (c) $S_{\text {sp }}$ and (d) $Z_{\text {sp }} T$ as functions of chemical potential $\mu$ under $V_{g}=8 \mathrm{~V}$ and $T=20 \mathrm{~K}$ for configuration I with different $N$.

ZGNR-polyacetylene-ZGNR junctions can work as good spin thermoelectric devices.

Finally, we emphasize that the calculated $Z_{\mathrm{sp}} T$ in Fig. 9 is only contributed by electrons, while the contribution from phonons is not included. This treatment is reasonable at low temperature. Theoretically, the figure of merit is determined by both thermal conductance of electrons $\kappa_{\mathrm{el}}$ and thermal conductance of phonons $\kappa_{\mathrm{ph}}$, where $\kappa=\kappa_{\mathrm{el}}+\kappa_{\mathrm{ph}}$. However, it has been indicated theoretically by Rameshti et al. [18] and experimentally by Zuev et al. [14] that $\kappa_{\mathrm{ph}}$ is very small and can be ignored in graphene when temperature is lower than $20 \mathrm{~K}$. In addition, the linear relationship of thermopower versus temperature was measured experimentally, which also suggests that phonon components to thermoelectric behavior in graphene can be neglected due to weak electron-phonon coupling [14].

\section{CONCLUSIONS}

In summary, we have investigated spin-resolved electric and thermoelectric transport behaviors in two kinds of ZGNRpolyacetylene-ZGNR magnetic molecular junctions by firstprinciples calculations. For the configuration I, thermally induced spin-up current is positive and spin-down current is negative, while for the configuration II, the situation is reversed. The opposite behaviors of $I_{\sigma}$ are mostly determined by the renormalized HOMO levels of polyacetylene chains under different connecting formations in both configurations. By adjusting the temperature gradient between two leads and the gate voltage applied in the molecular region, a pure spin current without charge current can be obtained, which is found in all the molecular junctions with different lengths. The direction of pure spin current in configuration I is opposite to that in configuration II at the same temperature gradient. At low temperatures, thermopower and thermal conductance show roughly linear behaviors as functions of temperature at the Fermi level. For configuration I, spinup thermopower is negative and spin-down thermopower is positive, while for configuration II, spin-up thermopower is positive and spin-down thermopower is negative. At specific energy, thermopower can be largely increased by adjusting the gate voltage, and furthermore a huge figure of merit $Z_{\mathrm{sp}} T$ can be obtained. Both kinds of graphene-based molecular magnetic junctions are good candidates of spin thermoelectric devices.

\section{ACKNOWLEDGMENTS}

This work was financially supported by grants from the National Natural Science Foundation of China (Grants No. 11304205, No. 11574217, No. 11374246, and No. 11504240) and Shenzhen Natural Science Foundation (JCYJ20130326111836781). J.W. acknowledges support from the University Grant Council (Contract No. AoE/P-04/08) of the Government of the HKSAR.
[1] Y. Kim, W. Jeong, K. Kim, W. Lee, and P. Reddy, Nat. Nanotechnol. 9, 881 (2014).
[2] A. Kirihara, K. Uchida, Y. Kajiwara, M. Ishida, Y. Nakamura, T. Manako, E. Saitoh, and S. Yorozu, Nat. Mater. 11, 686 (2012). 
[3] B. Wang, Y. X. Xing, L. H. Wan, Y. D. Wei, and J. Wang, Phys. Rev. B 71, 233406 (2005); B. Wang, Y. X. Xing, Y. D. Wei, L. H. Wan, and J. Wang, Carbon 43, 2786 (2005).

[4] E. Pop, S. Sinha, and K. E. Goodson, Proc. IEEE 94, 1587 (2006).

[5] S. A. Wolf, D. D. Awschalom, R. A. Buhrman, J. M. Daughton, S. von Molnar, M. L. Roukes, A. Y. Chtchelkanova, and D. M. Treger, Science 294, 1488 (2001).

[6] A. Fert, Rev. Mod. Phys. 80, 1517 (2008).

[7] K. Uchida, S. Takahashi, K. Harii, J. Ieda, W. Koshibae, K. Ando, S. Maekawa, and E. Saitoh, Nature (London) 455, 778 (2008)

[8] H. Adachi, K. Uchida, E. Saitoh, and S. Maekawa, Rep. Prog. Phys. 76, 036501 (2013).

[9] K. Uchida, J. Xiao, H. Adachi, J. Ohe, S. Takahashi, J. Ieda, T. Ota, Y. Kajiwara, H. Umezawa, H. Kawai, G. E. W. Bauer, S. Maekawa, and E. Saitoh, Nat. Mater. 9, 894 (2010).

[10] C. M. Jaworski, J. Yang, S. Mack, D. D. Awschalom, J. P. Heremans, and R. C. Myers, Nat. Mater. 9, 898 (2010).

[11] G. E. W. Bauer, E. Saitoh, and B. J. van Wees, Nat. Mater. 11, 391 (2012).

[12] K. S. Novoselov, V. I. Fal'ko, L. Colombo, P. R. Gellert, M. G. Schwab, and K. Kim, Nature (London) 490, 192 (2012).

[13] S. Das Sarma, S. Adam, E. H. Hwang, and E. Rossi, Rev. Mod. Phys. 83, 407 (2011).

[14] Y. M. Zuev, W. Chang, and P. Kim, Phys. Rev. Lett. 102, 096807 (2009).

[15] P. Wei, W. Z. Bao, Y. Pu, C. N. Lau, and J. Shi, Phys. Rev. Lett. 102, 166808 (2009).

[16] J. G. Checkelsky and N. P. Ong, Phys. Rev. B 80, 081413 (2009).

[17] F. Ghahari, H.-Y. Xie, T. Taniguchi, K. Watanabe, M. S. Foster, and P. Kim, Phys. Rev. Lett. 116, 136802 (2016).

[18] B. Z. Rameshti and A. G. Moghaddam, Phys. Rev. B 91, 155407 (2015).

[19] Z. Y. Zhao, X. C. Zhai, and G. J. Jin, Appl. Phys. Lett. 101, 083117 (2012).
[20] Y. Ni, K. L. Yao, H. H. Fu, G. Y. Gao, S. C. Zhu, and S. L. Wang, Sci. Rep. 3, 1380 (2013).

[21] M. G. Zeng, Y. P. Feng, and G. C. Liang, Nano Lett. 11, 1369 (2011); M. G. Zeng, H. Wen, and G. C. Liang, Nanoscale 5, 200 (2013).

[22] X. Chen, Y. Liu, B.-L. Gu, W. Duan, and F. Liu, Phys. Rev. B 90, 121403(R) (2014).

[23] L. Liang, E. Cruz-Silva, E. C. Girao, and V. Meunier, Phys. Rev. B 86, 115438 (2012).

[24] M. Saiz-Bretin, A. V. Malyshev, P. A. Orellana, and F. Dominguez-Adame, Phys. Rev. B 91, 085431 (2015).

[25] M. X. Zhai, X. F. Wang, P. Vasilopoulos, Y. S. Liu, Y. J. Dong, L. P. Zhou, Y. J. Jiang, and W. L. You, Nanoscale 6, 11121 (2014).

[26] K. Zberecki, M. Wierzbicki, J. Barnas, and R. Swirkowicz, Phys. Rev. B 88, 115404 (2013); K. Zberecki, R. Swirkowicz, and J. Barnas, ibid. 89, 165419 (2014).

[27] J. Taylor, H. Guo, and J. Wang, Phys. Rev. B 63, 245407 (2001); 63, 121104 (2001).

[28] M. Buttiker, Y. Imry, R. Landauer, and S. Pinhas, Phys. Rev. B 31, 6207 (1985).

[29] B. G. Wang, J. Wang, and H. Guo, J. Phys. Soc. Jpn. 70, 2645 (2001).

[30] T. Rejec, A. Ramsak, and J. H. Jefferson, Phys. Rev. B 65, 235301 (2002).

[31] W. Y. Kim and K. S. Kim, Nat. Nanotechnol. 3, 408 (2008).

[32] Y. W. Son, M. L. Cohen, and S. G. Louie, Nature (London) 444, 347 (2006).

[33] E. Rudberg, S. Pawel, and Y. Luo, Nano Lett. 7, 2211 (2007).

[34] D. Waldron, P. Haney, B. Larade, A. MacDonald, and H. Guo, Phys. Rev. Lett. 96, 166804 (2006); 97, 226802 (2006).

[35] D. R. Hamann, M. Schluter, and C. Chiang, Phys. Rev. Lett. 43, 1494 (1979).

[36] O. Gunnarsson and B. I. Lundqvist, Phys. Rev. B 13, 4274 (1976); 15, 6006(E) (1977).

[37] J. M. Soler, E. Artacho, J. D. Gale, A. Garcia, J. Junquera, P. Ordejon, and D. Sanchez-Portal, J. Phys.: Condens. Matter 14, 2745 (2002). 